\title{
TITULAÇÕES ESPECTROFOTOMÉTRICAS DE SISTEMAS ÁCIDO-BASE UTILIZANDO EXTRATO DE FLORES CONTENDO ANTOCIANINAS
}

\author{
Mônica Souza Cortes, Luiz Antônio Ramos e Éder Tadeu Gomes Cavalheiro* \\ Instituto de Química de São Carlos, Universidade de São Paulo, CP 780, 13560-970 São Carlos - SP, Brasil
}

Recebido em 3/1/06; aceito em 28/7/06; publicado na web em 26/3/07

\begin{abstract}
SPECTROPHOTOMETRIC TITRATIONS OF ACID-BASE SYSTEMS USING FLOWER EXTRACTS CONTAINIG ANTHOCYANINS. Considering the attraction of the students' attention by the changes in the colors of vegetable crude extracts caused by the variation of the $\mathrm{pH}$ of the medium, the use of these different colors in order to demonstrate principles of spectrophotometric acid-base titrations using the crude extracts as indicators is proposed. The experimental setup consisted of a simple spectrophotometer, a homemade flow cell and a pump to propel the fluids along the system. Students should be stimulated to choose the best wavelength to monitor the changes in color during the titration. Since the $\mathrm{pH}$ of the equivalence point depends on the system titrated, the wavelength must be properly chosen to follow these changes, demonstrating the importance of the correct choice of the indicator. When compared with the potentiometric results, errors as low as $2 \%$ could be found using Rhododendron simsii (azalea) or Tibouchina granulosa (Glory tree, quaresmeira) as sources of the crude extracts.
\end{abstract}

Keywords: acid-base chemistry; instrumental methods; spectrophotometric titrations.

\section{INTRODUÇÃO}

O uso de corantes naturais no ensino médio e superior, principalmente de química geral e analítica, tem sido proposto freqüentemente. As principais vantagens são relacionadas com a atração do interesse dos estudantes para o conteúdo abordado, devido à coloração das espécies químicas contidas nos tecidos vegetais e suas mudanças de cor em função do $\mathrm{pH}^{1,2}$. Some-se a isto o fato de usar algo presente no cotidiano dos estudantes para esta finalidade, ligando a química ao seu dia-a-dia.

Neste contexto, os corantes foram propostos para demonstração de conceitos de $\mathrm{pH}^{1-3}$, como indicadores em titulações ácido-base, com detecção visual do ponto final ${ }^{4}$, para demonstração de fundamentos de cromatografia em camada delgada ${ }^{5}$ e aplicação em métodos óticos de análise ${ }^{6}$, de princípios de análise em fluxo ${ }^{7}$ e na determinação de sulfito em vinhos ${ }^{8}$ e de nitrito em águas 9 .

As mudanças na coloração de extratos de tecidos vegetais foram abordadas inicialmente por Geissman ${ }^{10}$, que as atribuiu às antocianinas. As antocianinas são as formas glicosiladas das antocianidinas que, por sua vez, são compostos da classe dos flavonóides e que conferem coloração azul ou vermelha aos tecidos vegetais ${ }^{1,10}$.

As transformações estruturais que provocam tais mudanças de cor em função do $\mathrm{pH}$ foram também descritas previamente por Brouillard e colaboradores ${ }^{11,12}$ e por Epp $^{13}$.

As estrutras químicas das antocianinas e as mudanças estruturais, que ocorrem com a variação do $\mathrm{pH}$ e provocam as mudanças de cor, podem ser encontradas nas referências citadas acima. Uma discussão mais aprofundada sobre esta classe interessante de flavonóides pode ser encontrada no trabalho de Timberlake e Bridle ${ }^{14}$.

Neste artigo, propõe-se o aproveitamento da mudança de cor de extratos brutos de espécies vegetais contendo antocianinas para a detecção espectrofotométrica de pontos finais de titulações ácido-base. Conceitos importantes como detecção de ponto final, cores complementares, fundamentos de espectroscopia, Lei de Beer,

*e-mail: cavalheiro@iqsc.usp.br hidrólise e seu efeito no $\mathrm{pH}$ do ponto final e escolha de indicadores, entre outros, podem ser abordados com a vantagem de atrair a atenção dos alunos pelo uso dos corantes naturais.

\section{FUNDAMENTAÇÃO}

\section{Titulações espectrofotométricas ${ }^{15-18}$}

Medidas espectrofotométricas, ou fotométricas, podem ser utilizadas na detecção do ponto final de titulações, desde que haja variação na coloração das espécies envolvidas, ou de um indicador, no ponto final.

No caso de titulações ácido-base, normalmente adiciona-se a base ao ácido e mede-se a absorbância do sistema em função do volume adicionado, em um comprimento de onda $(\lambda)$ pré-estabelecido. O $\lambda$ deve ser tal, que represente o máximo de absorção do ácido ou da base, caso sejam coloridos, ou do indicador cuja cor mude no ponto final. A absorção de radiação também pode ocorrer na região do ultravioleta.

Assim, o resultado de uma titulação espectrofotométrica é um gráfico que representa a variação da absorbância, no $\lambda_{\text {max }}$ de absorção da espécie que está sendo monitorada, em função do volume do titulante adicionado.

Esse gráfico pode apresentar formas diferentes dependendo das características da absorção de radiação pelas espécies envolvidas, no comprimento de onda monitorado. A Figura 1 mostra tais possibilidades, como a reação de titulado que não absorve com titulante colorido, gerando produto que também não absorve no $\lambda$ monitorado (Figura 1a); formação de produto colorido a partir de reagentes que não absorvem neste comprimento de onda (Figura 1b), além de várias outras possibilidades. Nesta figura, $\boldsymbol{\varepsilon}$ representa o coeficiente de absortividade molar da espécie, enquanto $\boldsymbol{a}, \boldsymbol{t}$ e $\boldsymbol{p}$ representam o titulado (amostra), o titulante e o produto da reação, respectivamente.

A absorbância deve ser corrigida a cada adição do titulante pois, segundo a Lei de Lambert-Beer, é proporcional à concentração das espécies presentes e a adição do titulante provoca diluição do sistema. A correção é feita pela Equação $1^{16}$ 


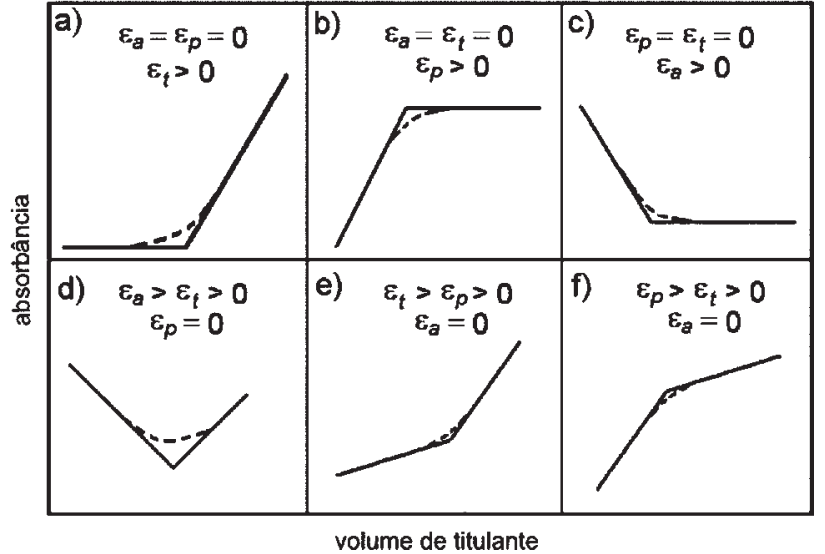

Figura 1. Curvas típicas de titulações fotométricas. Adaptado da ref. 15

$$
A_{\text {corr }}=A_{\text {lida }} \times\left(\frac{V+v}{V}\right)
$$

na qual $\boldsymbol{A}_{\text {corr }}$ é a absorbância corrigida, $\boldsymbol{A}_{\text {lida }}$ é a absorbância obtida em cada ponto, $\boldsymbol{V}$ o volume inicial e $\boldsymbol{v}$ o incremento de volume a cada ponto.

A determinação do ponto final pode ser feita extrapolando-se os segmentos lineares na região do ponto final. A intersecção destas porções indica a região da mudança de comportamento do indicador, refletindo o ponto final.

A literatura apresenta inúmeros exemplos do uso destas titulações, demonstrando sua importância. Exemplos deste tipo de procedimento incluem revisões bibliográficas ${ }^{19,20}$, descrição de fundamentos e instrumentação ${ }^{21-39}$, determinação de metais usando reações de complexação ${ }^{40-51}$, reações em meio não aquoso ${ }^{52-55}$. Além destes, há vários outros trabalhos envolvendo este tipo de titulações, todos apresentando potencialidades para aplicações didáticas.

Trabalhos envolvendo experimentos didáticos também podem ser encontrados. Destaca-se o uso de dispositivos para detecção do ponto final ${ }^{56}$ e deteção simultânea de pontos finais de titulações colométricas e fotométricas ${ }^{57}$.

A principal vantagem é que os dados experimentais são obtidos fora da região de equivalência, favorecendo sistemas cujo equilíbrio não esteja totalmente deslocado para a formação de produtos, como ocorre na potenciometria, ou no caso do uso de indicadores visuais, o que permite titular sistemas relativamente diluídos ${ }^{15}$. A escolha adequada do comprimento de onda da espécie a ser monitorada permite realizar a titulação na presença de outras espécies coloridas.

É importante ressaltar que, apesar da simplicidade instrumental requerida, precisão e riqueza dos aspectos conceituais envolvidos, relativamente pouco se explora da fotometria em relação às titulações, em aulas práticas.

\section{PARTE EXPERIMENTAL}

\section{Reagentes e soluções}

Água destilada e desionizada foi utilizada no preparo das soluções. Todos os reagentes foram de grau analítico (PA) e utilizados sem purificação prévia. Soluções tampão universal foram preparadas conforme descrito por Perrin e Dempsey ${ }^{58}$ : tetraborato de sódio $5,7 \mathrm{mmol} \mathrm{L}^{-1}$; fosfato dissódico monoácido $20 \mathrm{mmol} \mathrm{L}^{-1} \mathrm{e}$ citrato trissódico $13 \mathrm{mmol} \mathrm{L}^{-1}$. Foram feitas soluções para $\mathrm{pH}$ 2,0; 5,0; 6,0...; 9,0, ajustando-se com ácido clorídrico ou hidróxido de sódio. Com esta composição, a força iônica das soluções era de aproxi- madamente $0,1 \mathrm{~mol} \mathrm{~L}^{-1}$. Diariamente, antes de iniciar os experimentos, determinava-se o $\mathrm{pH}$ destas soluções empregando-se eletrodo de vidro e um $\mathrm{pH}$-metro com resolução de 0,01 unidades de $\mathrm{pH}$. Previamente o $\mathrm{pH}$-metro era calibrado com soluções tampão da Merck ( $\mathrm{pH} \mathrm{4,0} \mathrm{e} \mathrm{10,0).} \mathrm{Estas} \mathrm{soluções} \mathrm{foram} \mathrm{utilizadas} \mathrm{para}$ controle de $\mathrm{pH}$ nas medidas de absorção na região do visível.

Soluções de ácido clorídrico $(\mathrm{HCl})$, ácido acético (HAc), hidróxido de sódio $(\mathrm{NaOH})$ e hidróxido de amônio $\left(\mathrm{NH}_{4} \mathrm{OH}\right)$ foram preparadas, em concentrações próximas de $0,1 \mathrm{~mol} \mathrm{~L}^{-1}$ e a seguir padronizadas $^{59}$.

\section{Equipamentos}

Todas as medidas podem ser realizadas em um espectrofotômetro convencional capaz de realizar leituras na região do visível. No caso deste trabalho, os espectros de absorção das soluções contendo uma concentração de corante em diferentes $\mathrm{pH}$ foram obtidos com auxílio de espectrofotômetro MultiSpec 1501 (Shimadzu, Japão), usando-se cubetas de polimetacrilato com caminho ótico de $1,0 \mathrm{~cm}$.

As titulações espectrofotométricas foram realizadas usando-se espectrofotômetro Genesis-20 (Spectronics, EUA), sendo as soluções transferidas com auxílio de bomba peristáltica IPC-4 (Ismatec, Suíça) e uma célula de fluxo preparada nas oficinas do IQSC/USP.

Alternativamente, um fotômetro capaz de monitorar o $\lambda_{\max }$ da espécie a ser acompanhada também pode ser utilizado, simplificando a instrumentação.

\section{Preparação dos extratos brutos}

Dependendo da disponibilidade do tempo, os estudantes podem preparar os extratos brutos de flores antes do experimento ou, alternativamente, a equipe instrutora pode prepará-los e fornecer para os estudantes no início da aula. Os extratos podem ser preparados com as pétalas recém-colhidas ou congeladas por até 1 mês. Para obtenção do extrato bruto, foram utilizadas aproximadamente 25 g de pétalas de Rhododendron simsii (azaléa), ou Tibouchina granulosa (quaresmeira), imersas em $100 \mathrm{~mL}$ de etanol. O tempo de extração estabelecido foi de $48 \mathrm{~h}$, mantendo-se o material em frasco de vidro envolvido em papel alumínio e à temperatura ambiente $^{1,4}$. Após filtração, o extrato foi evaporado em evaporador rotatório até obtenção de um líquido viscoso.

Alternativamente, flores de outras espécies que contenham antocianinas ${ }^{1,4,7}$ podem ser empregadas como fonte dos indicadores naturais. Sugere-se determinar os espectros de absorção em função do $\mathrm{pH}$, para a espécie escolhida, pois a composição de corantes no extrato pode variar dependendo da espécie.

\section{Medidas espectrofotométricas}

Obtenção do espectro de absorção e determinação do comprimento de onda no máximo de absorção para os extratos

Espectros das soluções contendo os corantes vegetais foram obtidos na região do visível, em diferentes pHs, para determinação dos comprimentos de onda dos máximos de absorção e para avaliar as mudanças espectrais em função do $\mathrm{pH}$. Para obter os espectros de absorção sugere-se usar uma solução contendo $100 \mathrm{mg}$ de extrato bruto dissolvidos em 10,0 mL de água $\left(10 \mathrm{~g} \mathrm{~L}^{-1}\right)$. A partir desta solução, sugere-se que sejam preparadas soluções de trabalho em pH 2,0; 5,0; 6,0; 7,0; 8,0 e 9,0; adicionando-se $1,0 \mathrm{~mL}$ da solução de extrato bruto a um balão volumétrico com capacidade para $10,0 \mathrm{~mL}$. O volume deve ser completado com solução tampão universal, no $\mathrm{pH}$ desejado que foi previamente ajustado. 
Recomenda-se que o comprimento de onda seja escolhido de tal maneira que uma variação marcante na absorbância ocorra no ponto de final. Para cada sistema ácido forte - base forte, ácido fraco - base fraca, etc., deve-se escolher um comprimento de onda em que a mudança de cor do extrato ocorra próximo ao $\mathrm{pH}$ de viragem.

\section{Titulações espectrofotométricas}

As titulações espectrofotométricas foram desenvolvidas em um sistema representado na Figura 2. Neste caso, uma tubulação em polietileno com diâmetro interno de $0,8 \mathrm{~mm}$ e $80 \mathrm{~cm}$ de comprimento (Fiotubo, Brasil) foi usada para transportar o líquido até uma cela de fluxo, inserida no espectrofotômetro e retorná-lo até o vaso de titulação, para cada fração de volume adicionado. $\mathrm{O}$ vaso de titulação continha um agitador magnético e uma bomba peristáltica, que foi usada na propulsão dos fluidos.

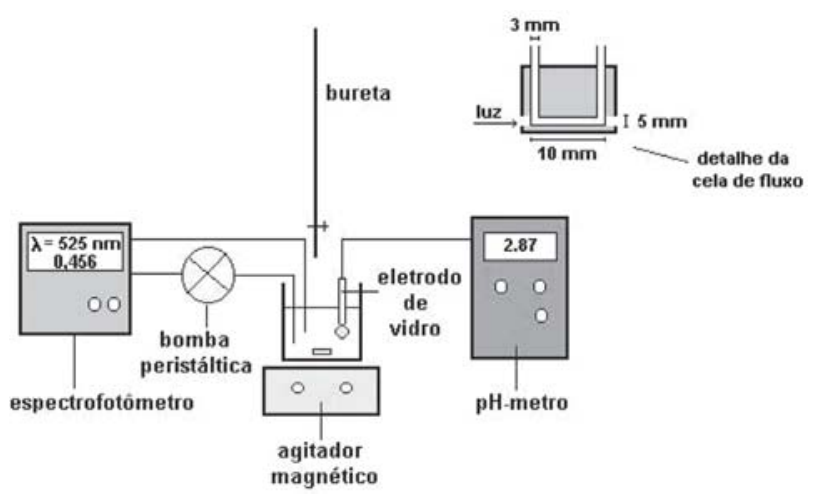

Figura 2. Representação esquemática do sistema de titulação com detecção simultânea espectofotométrica e potenciométrica

Após a adição do volume desejado de titulante, o sistema foi agitado com auxílio de agitador magnético e bombeado pelo sistema por $15 \mathrm{~s}$, a uma vazão de $10 \mathrm{~mL} \mathrm{~min}{ }^{-1}$. A vazão foi interrompida por desligamento da bomba e fez-se a leitura de absorbância após estabilização. A bomba foi então religada até que o volume contido no sistema de circulação fosse transferido para o vaso de titulação, retirando-se o tubo de entrada da solução. Fez-se nova adição de titulante, reiniciando-se o processo.

Para comparação dos resultados, mediu-se o pH da solução após cada incremento obtendo-se, simultaneamente, uma titulação potenciométrica.

\section{RESULTADOS E DISCUSSÃO}

Os resultados descritos a seguir foram obtidos pelos autores deste trabalho. Uma aplicação didática deveria seguir os mesmos passos: os alunos preparariam os extratos brutos em diferentes $\mathrm{pHs}$; usando um espectrofotômetro, obteriam espectros das soluções contendo os extratos brutos para escolha do melhor comprimento de onda considerando as variações espectrais em função do $\mathrm{pH}$; finalmente, após montar o sistema, fariam as titulações e tratariam os resultados obtidos.

\section{Espectros de absorção na região do visível dos extratos brutos em diferentes pHs}

Espectros de absorção típicos das soluções contendo $10 \mathrm{~g} \mathrm{~L}^{-1}$ dos extratos brutos de Rhododendron simsii (azaléa, Figura 3a) e de Tibouchina granulosa (quaresmeira, Figura 3b), em diferentes pHs são apresentados na Figura 3.
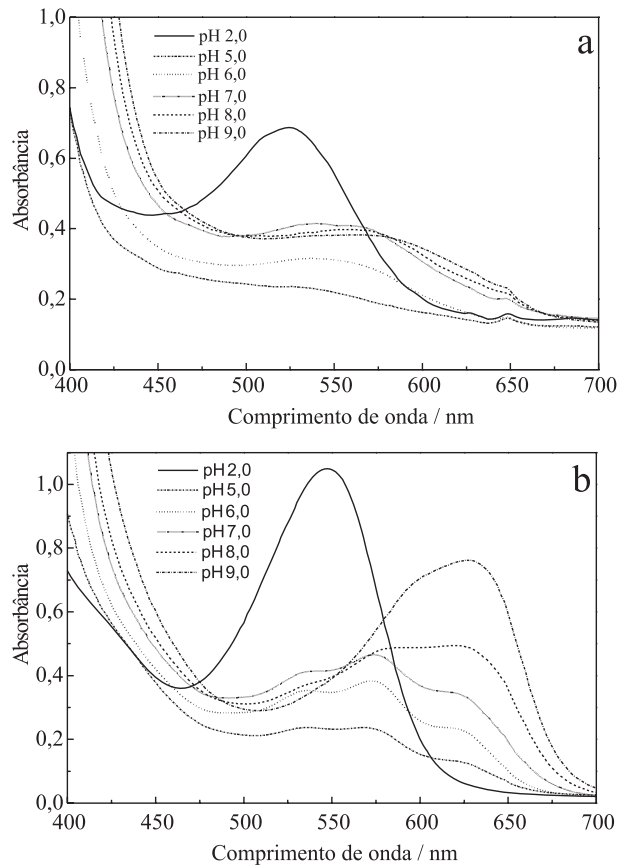

Figura 3. Espectros de absorção dos extratos brutos: (a) Rhododendron simsii (azaléa, $10 \mathrm{~g} \mathrm{~L}^{-1}$ ), (b) Tibouchina granulosa (quaresmeira, $10 \mathrm{~g} \mathrm{~L}^{-1}$ ) em diferentes $\mathrm{pHs}$

Pode-se observar, na Figura 3, que o comprimento de onda do máximo de absorção se desloca com a variação do $\mathrm{pH}$ para os dois extratos brutos. Quimicamente, os deslocamentos do comprimento de onda do máximo de absorção estão relacionados com as diferenças nas estruturas das antocianinas provocadas pela mudança na acidez do meio, resultando em espectros de absorção diferentes ${ }^{1,2,11-13}$.

De maneira geral, observando-se visualmente as soluções, podese dizer que, em meio ácido $(\mathrm{pH}=2,0)$, predomina coloração vermelha para os dois extratos. Aumentando-se o pH, a cor muda de vermelho para verde em $\mathrm{pH} 8,0$. Em valores de $\mathrm{pH}$ mais elevados observa-se uma coloração amarela, com absorção intensa no ultravioleta (UV). Quando se mantém por tempo prolongado o extrato em meio básico, a cor amarela é irreversível. Este fato pode estar relacionado com a formação de chalcona ${ }^{1}$.

No caso da Rhododendron simsii (azaléa), monitorando-se a absorbância em $525 \mathrm{~nm}$, observa-se que esta diminui quando se aumenta o $\mathrm{pH}$ de 2,0 para 5,0 (de vermelho para quase-incolor), aumenta entre 6,0 e 7,0 (verde) e assume um valor constante a partir de $\mathrm{pH}$ 7,0 (azul), sugerindo que este seja um bom indicador para sistemas com $\mathrm{pH}$ neutro no ponto final, como pode ser observado na Figura 3. Acima de pH 10,0 a solução fica amarela, devido à presença das chalconas.

O extrato de Tibouchina granulosa (quaresmeira) apresentou resultados semelhantes em $550 \mathrm{~nm}$. Entretanto, bandas com maior intensidade foram observadas em torno de $630 \mathrm{~nm}$, sendo a absorbância crescente com o aumento do pH.

A partir dos espectros é possível escolher um comprimento de onda para acompanhar a titulação de acordo com o sistema ácidobase a ser titulado, como descrito a seguir.

\section{Escolha do melhor comprimento de onda para detecção espectrofotométrica do ponto final}

Durante o curso de uma titulação ácido-base, o pH do meio varia dependendo da ordem de adição dos reagentes, sua concentração, quem é o titulado e quem é o titulante e, também, de acordo 
com a extensão da ionização, "força", dos ácidos e bases envolvi$\operatorname{dos}^{15,59,60}$.

Conseqüentemente, o $\mathrm{pH}$ do ponto final é, também, dependente das espécies envolvidas e suas concentrações. Pode-se considerar as seguintes situações:

ácido forte titulado com base forte: neste caso, a reação de neutralização resulta em um sal que não apresenta hidrólise, nem de seu cátion nem de seu ânion e, portanto, o $\mathrm{pH}$ do ponto final é devido à constante de ionização da água: $\mathrm{pH}=7,0$ a $25{ }^{\circ} \mathrm{C}$.

Para a azaléa, Figura $3 \mathrm{a}$, no $\lambda=525 \mathrm{~nm}$ a absorbância cai até pH 5,0 e acima de pH 8,0 torna-se aproximadamente constante, sugerindo que o uso deste comprimento de onda seja adequado para monitoramento deste tipo de titulação.

ácido fraco titulado com base forte: forma-se um sal cujo ânion é a base conjugada de um ácido fraco e um cátion que é ácido conjugado de uma base forte. Só o ânion tem tendência à hidrólise e o pH do ponto final será regido pela quantidade deste ânion presente, sua concentração e pela constante de hidrólise deste ânion, segundo a reação:

$\mathrm{B}+\mathrm{H}_{2} \mathrm{O} \rightleftharpoons \mathrm{BH}^{+}+\mathrm{OH}^{-}$

Segundo esta reação formam-se íons $\mathrm{OH}^{-}$, que conferem caráter básico à solução no ponto final.

Em $\lambda=525 \mathrm{~nm}$ para os dois extratos observa-se que a absorbância diminui entre pH 2,0 e 5,0, aumenta entre pH 5,0 e 7,0 e se torna constante acima de $\mathrm{pH} 8,0$, podendo-se monitorar este tipo de sistema com o indicador natural, neste comprimento de onda. base fraca titulada com ácido forte: analogamente, neste caso, temse a hidrólise do cátion, ácido conjugado da base fraca:

$\mathrm{BH}^{+}+\mathrm{H}_{2} \mathrm{O} \rightleftharpoons \mathrm{B}+\mathrm{H}_{3} \mathrm{O}^{+}$

Aqui a hidrólise gera íons $\mathrm{H}_{3} \mathrm{O}^{+}$, que conferem caráter ácido à solução resultante no ponto final.

Em $\lambda=625 \mathrm{~nm}$ observa-se a diminuição da absorbância entre $\mathrm{pH} 2,0$ e 5,0, um aumento significativo entre $\mathrm{pH}$ 5,0 e 7,0 e um aumento mais suave acima de $\mathrm{pH} 7,0$, para a azaléa, que permite monitorar este tipo de sistema em $\lambda=625 \mathrm{~nm}$.

base fraca titulada com ácido fraco: os dois íons componentes do sal podem apresentar hidrólise e deve-se considerar as constantes de dissociação ácida, $\mathrm{K}_{\mathrm{a}}$, e de dissociação básica, $\mathrm{K}_{\mathrm{b}}$, além das concentrações das espécies para estimar o $\mathrm{pH}$ do ponto final. No caso de: $\mathrm{K}_{\mathrm{a}}>\mathrm{K}_{\mathrm{b}}$ - resulta solução ácida; $\mathrm{K}_{\mathrm{a}}<\mathrm{K}_{\mathrm{b}}$ - resulta solução básica; $K_{a}=K_{b}$ - resulta solução neutra.

Desta forma, na escolha do indicador é preciso levar em conta todas estas considerações para que se cometa o menor erro possível na determinação do ponto final. Sendo este um caso que envolve muitas variáveis, não foi usado nos exemplos práticos.

\section{Resultados obtidos nas titulações espectrofotométricas para diferentes sistemas ácido-base}

A seguir são apresentados alguns exemplos de curvas de titulações para diferentes sistemas ácido-base, obtidas usando-se o extrato bruto de Rhododendron simsii (azaléa, $10 \mathrm{~g} \mathrm{~L}^{-1}$ ) como indicador ácido-base. Os pontos finais foram determinados pela primeira derivada da curva potenciométrica e construindo-se curvas de absorbância corrigida versus volume de base adicionado, fazendose as devidas extrapolações dos ramos destas curvas, como se descreve a seguir, para cada caso.

Na Figura 4 são apresentadas as curvas das respostas espectrofotométrica (Figura 4a) e potenciométrica (Figura 4b) obtidas na titulação de $\mathrm{HCl}$ com $\mathrm{NaOH}$. Como se pode observar, os valores obtidos na detecção espectrofotométrica monitorada no $\lambda 525 \mathrm{~nm}$, usando como indicador extrato bruto de azaléa, foram comparados com os valores obtidos na detecção potenciométrica utilizando um eletrodo de vidro combinado.
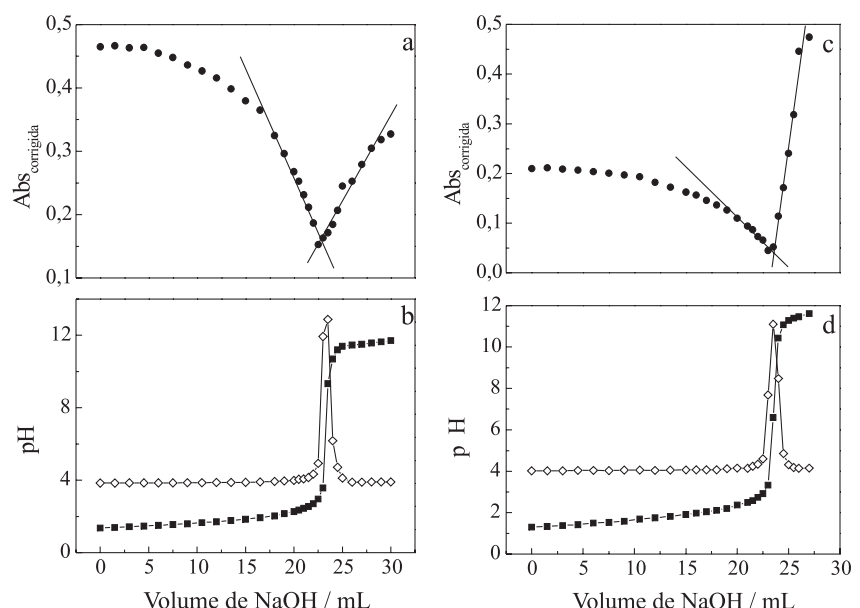

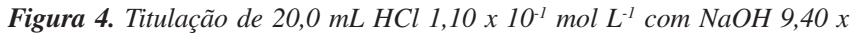
$10^{-2}$ mol $L^{-1}$ com detecção espectrofotométrica $(a)$ e potenciométrica $(b)$. Volume de extrato bruto $\left(10 \mathrm{~g} \mathrm{~L}^{-1}\right)=2,0 \mathrm{~mL}, \lambda=525 \mathrm{~nm}$ para Rhododendron simsii. No caso da Tibouchina granulosa (quaresmeira): (c) curva espectrofotométrica com detecção em $625 \mathrm{~nm}$ e (d) curva potenciométrica

Como sugerem os espectros da Figura 3a, a absorbância diminui com o consumo de íons $\mathrm{H}_{3} \mathrm{O}^{+}$, até $\mathrm{pH} \approx 5,0$. Com novas adições de $\mathrm{NaOH}$, a absorbância volta a aumentar. $\mathrm{O}$ menor valor na curva de absorbância corrigida em função do volume de $\mathrm{NaOH}$ adicionado foi considerado como sendo o volume do final para cálculo.

$\mathrm{O}$ erro pode ser minimizado diminuindo-se o incremento do volume adicionado na região do salto de $\mathrm{pH}$. Um exemplo típico adotando este procedimento foi realizado usando o extrato bruto de Tibouchina granulosa (quaresmeira), apresentado na Figura 4c curva espectrofotométrica (com detecção em $625 \mathrm{~nm}$ ), o qual também foi comparado com o resultado potenciométrico apresentado na Figura 4d.

Na Figura 5 são apresentadas as curvas das respostas espectrofotométrica (Figura 5a) e potenciométrica (Figura 5b) obtidas na titulação de $\mathrm{HAc}$ com $\mathrm{NaOH}$. Pode-se observar que o perfil da curva

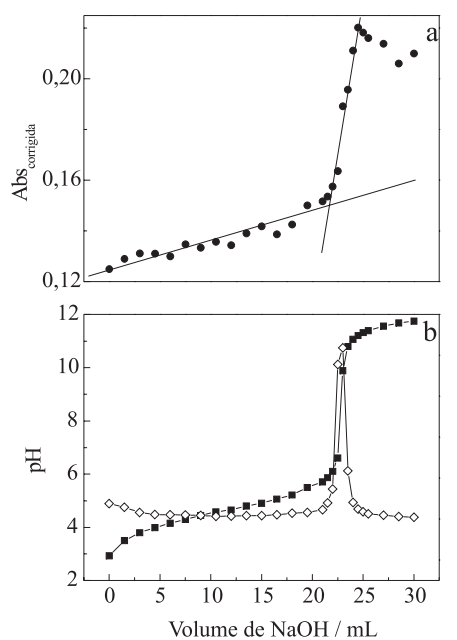

Figura 5. Titulação de 20,0 mL HAc 1,08 $\times 10^{-1} \mathrm{~mol} \mathrm{~L}^{-1} \mathrm{com} \mathrm{NaOH} \mathrm{9,40x}$ $10^{-2} \mathrm{~mol} \mathrm{~L}^{-1}$ com detecção espectrofotométrica (a) e potenciométrica $(b)$. Volume de extrato bruto $\left(10 \mathrm{~g} \mathrm{~L}^{-1}\right)=2,0 \mathrm{~mL}, \lambda=520 \mathrm{~nm}$ 
da absorbância monitorada em $\lambda 525 \mathrm{~nm}$ muda, pois a acidez do meio é menor no início da titulação, devido à pequena extensão de ionização do ácido fraco, quando comparado ao $\mathrm{HCl}$. Após a adição do titulante ocorre a formação de um sistema tampão, que impede variações bruscas de $\mathrm{pH}$ até próximo do ponto final ${ }^{15}$.

Inicialmente a absorbância varia lentamente para valores menores, seguida de um aumento súbito a partir de $\mathrm{pH}$ 6,0, tendendo a uma estabilização acima de $\mathrm{pH}$ 10,0. O ponto final foi determinado pela intersecção da fração linear descendente com a reta representada pelo salto de absorbância entre pH 4,0 e 10,0.

Na Figura 6 são apresentadas as curvas das respostas espectrofotométrica (Figura 6a) e potenciométrica (Figura 6b) obtidas na titulação de $\mathrm{HCl}$ com $\mathrm{NH}_{4} \mathrm{OH}$. Neste exemplo de titulação foi necessário escolher $\lambda=625 \mathrm{~nm}$, para poder observar a viragem na região ácida, de acordo com os espectros da Figura 3, os quais mostram que, neste caso, a absorbância diminui até pH 5,0, aumentando bruscamente até pH 9,0.

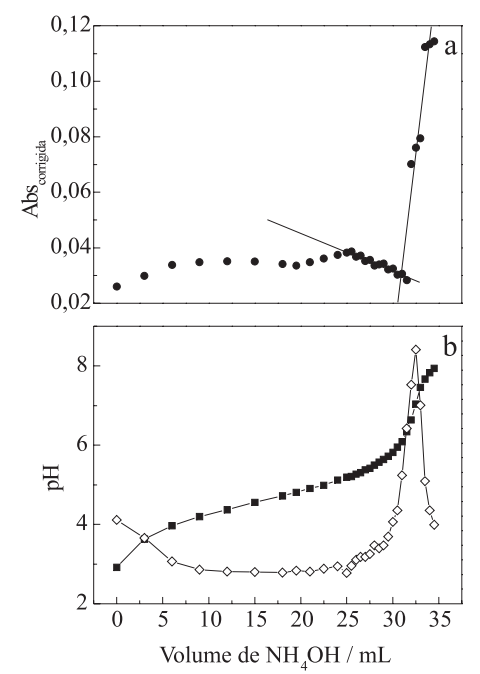

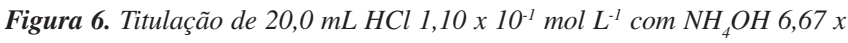
$10^{-2} \mathrm{~mol} \mathrm{~L}^{-1}$ com detecção espectrofotométrica (a) e potenciométrica (b). Volume de extrato bruto $\left(10 \mathrm{~g} \mathrm{~L}^{-1}\right)=2,0 \mathrm{~mL}, \lambda=620 \mathrm{~nm}$
Na curva de titulação, a acidez do meio mantém a absorbância aproximadamente constante até um volume adicionado de base de aproximadamente $25,0 \mathrm{~mL}$, quando começa a diminuir até $32,0 \mathrm{~mL}$, com $\mathrm{pH}$ próximo a 5,0. Ocorre, então, um aumento brusco na absorbância até que o $\mathrm{pH}$ atinja um valor próximo a 8,0. O ponto final foi obtido pela intersecção da reta, representada pela fração descendente e uma linha reta traçada unindo as extremidades do salto de absorbância. O ponto final coincidiu com o mínimo da curva.

Na Tabela 1 são apresentados os resultados típicos obtidos nas titulações espectrofotométricas utilizando o extrato bruto de Rhododendron simsii (azaléa) comparados com os valores obtidos nas titulações potenciométricas, assim como os respectivos erros relativos.

Os resultados obtidos usando o método espectrofotométrico proposto são bastante próximos daqueles obtidos usando o método potenciométrico. A única exceção foi para o sistema $\mathrm{HCl}$ versus $\mathrm{NaOH}$, em que erro relativo foi da ordem de $\pm 2 \%$. O resultado poder ser melhorado obtendo-se mais pontos próximos ao volume do ponto final.

Os resultados obtidos com o extrato de Tibouchina granulosa (quaresmeira, $10 \mathrm{~g} \mathrm{~L}^{-1}$ ) foram semelhantes. Na Tabela 2 são apresentados os resultados quantitativos obtidos e sua comparação com aqueles das Figuras 4-6 e da Tabela 1. Os pontos finais foram determinados pela primeira derivada da curva potenciométrica e por procedimentos específicos para curva de absorbância como descritos no texto, como no caso anterior da Rododhendron simsii (azaléa).

Na Tabela 2 observa-se que os erros em relação ao método potenciométrico são menores que $1 \%$ em todos os casos, mostrando que os corantes naturais podem ser usados como indicadores em titulações espectrofotométricas, desde que escolhidos comprimentos de onda adequados. A melhora nos resultados em relação ao sistema $\mathrm{HCl} \times \mathrm{NaOH}$ pode ser atribuída à introdução de mais um ponto na região do salto de $\mathrm{pH}$ e pode ser um interessante exemplo didático.

\section{CONSIDERAÇÕES FINAIS}

Os resultados demonstram que é possível usar extratos brutos de flores contendo antocianinas como indicadores nas determinações de pontos finais em titulações espectrofotométricas. Entretanto, é preciso salientar a necessidade de escolher adequadamente

Tabela 1. Resultados obtidos nas titulações espectrofotométricas para os diferentes sistemas ácido-base, usando como indicador o extrato bruto de Rhododendron simsii (azaléa) comparados com os valores obtidos nas titulações potenciométricas

\begin{tabular}{|c|c|c|c|c|c|c|}
\hline \multirow[t]{2}{*}{ Sistema } & \multirow[t]{2}{*}{$\lambda / \mathrm{nm}$} & \multicolumn{3}{|c|}{ Volume do ponto final* / mL } & \multirow[t]{2}{*}{ Erro $_{1} / \%$} & \multirow[t]{2}{*}{ Erro $_{2} / \%$} \\
\hline & & Potenciometria & Espectrofotometria & Calc. & & \\
\hline $\mathrm{HCl} \times \mathrm{NaOH}$ & 525 & $23,5 \pm 0,0$ & $23,0 \pm 0,0$ & 23,4 & 2 & 2 \\
\hline $\mathrm{HAc} \times \mathrm{NaOH}$ & 520 & $23,0 \pm 0,1$ & $23,0 \pm 0,3$ & 22,9 & 0,4 & 0 \\
\hline $\mathrm{HCl} \times \mathrm{NH}_{4} \mathrm{OH}$ & 620 & $33,0 \pm 0,7$ & $33,1 \pm 0,1$ & 32,8 & 0,3 & 0,9 \\
\hline
\end{tabular}

*média de três determinações; $\mid$ Erro $_{1} \mid \%=(($ espectrofotométrico - potenciométrico $) /$ potenciométrico $)$ x $100 ; \mid$ Erro $2 \mid \%=(($ espectrofotométrico - calculado) / calculado) x 100

Tabela 2. Resultados obtidos nas titulações espectrofotométricas para deferentes sistemas ácido-base usando como indicador o extrato bruto de Tibouchina granulosa (quaresmeira) comparados com os valores obtidos nas titulações potenciométricas

\begin{tabular}{lcccccc}
\hline Sistema & $\lambda / \mathrm{nm}$ & \multicolumn{2}{c}{ Volume do ponto final*/mL } & $\mid$ Erro $_{1} \mid / \%$ & $\mid$ Erro $_{2} \mid / \%$ \\
& & Potenciometria & Espectrofotometria & Calc. & \\
\hline $\mathrm{HCl} \times \mathrm{NaOH}$ & 525 & $23,3 \pm 0,2$ & $23,5 \pm 0,0$ & 23,8 & 0,5 & 2 \\
$\mathrm{HAc} \times \mathrm{NaOH}$ & 625 & $22,0 \pm 0,1$ & $22,2 \pm 0,6$ & 22,0 & 0,7 & 1 \\
$\mathrm{HCl} \times \mathrm{NH}_{4} \mathrm{OH}$ & 550 & $21,6 \pm 0,2$ & $21,4 \pm 0,1$ & 21,6 & 0,6 & 1 \\
\hline
\end{tabular}

*media de três determinações; $\mathrm{NaOH}=8,90 \times 10^{-2} \mathrm{~mol} \mathrm{~L}^{-1}, \mathrm{NH}_{4} \mathrm{OH}=9,80 \times 10^{-2} \mathrm{~mol} \mathrm{~L}^{-1}, \mathrm{HCl}=10,6 \times 10^{-2} \mathrm{~mol} \mathrm{~L}^{-1}, \mathrm{HAc}=9,80 \times 10^{-2} \mathrm{~mol} \mathrm{~L}^{-1}$; $\mid$ Erro $_{1} \mid \%=(($ espectrofotométrico - potenciométrico $) /$ potenciométrico $) \times 100 ; \mid$ Erro $_{2} \mid \%=(($ espectrofotométrico - calculado $) /$ calculado $) \times 100$ 
o comprimento de onda de monitoramento, considerando-se os comportamentos dos diferentes sistemas e o $\mathrm{pH}$ dos pontos finais, que são dependentes da ocorrência ou não de hidrólise dos sais formados nas reações de neutralização.

Com base nas variações espectrais observadas para os extratos brutos, é possível avaliar o melhor comprimento de onda para cada caso. Esta demonstração fica bastante evidente e a torna bastante útil do ponto de vista didático. Erros experimentais também podem ser discutidos em relação à escolha do indicador e sua correta aplicação.

\section{AGRADECIMENTOS}

À Dra. C. C. S. Cavalheiro (IQSC-USP), pela colaboração e sugestões, à FAPESP pelo financiamento (Proc. 04/00407-4) e ao Programa PROCONTES/USP.

\section{REFERÊNCIAS}

1. Couto, A. B.; Ramos, L. A.; Cavalheiro, E. T. G.; Quim. Nova 1998, 21, 221 .

2. Terci, D. B. L.; Rossi, A. V.; Quim. Nova 2002, 25, 684.

3. Soares, M. H. F. B.; Boldrin-Silva, M. V.; Cavalheiro, E. T. G.; Eclet. Quim. 2001, 26, 225.

4. Soares, M. H. F. B.; Antunes, P. A.; Cavalheiro, E. T. G.; Quim. Nova 2001, 24, 408.

5. Okumura, F.; Soares, M. H. F. B.; Cavalheiro, E. T. G.; Quim. Nova 2002, 25,680 .

6. Soares, M. H. F. B.; Couto, A. B.; Ramos, L. A.; Cavalheiro, E. T. G.; Book of Abstracts, XI Euroanalysis, Lisbon, 2000.

7. Prieto, K. R.; Ramos, L. A.; Cavalheiro, C. C. S.; Cavalheiro, E. T. G.; J. Chem. Educ. 2005, 82, 1815.

8. Soares, M. H. F. B.; Ramos, L. A.; Cavalheiro, E. T. G.; J. Chem. Educ. 2002, 79, 1111.

9. Ramos, L. A.; Carla, C. C. S.; Cavalheiro, E. T. G.; Quim. Nova 2006, 29, 1114.

10. Geissman, T. A.; J. Chem. Educ. 1941, 18, 108

11. Brouillard, R.; Dubois, J. E.; J. Am. Chem. Soc. 1977, 99, 1359.

12. Brouillard, R.; Delaporte, B.; J. Am. Chem. Soc. 1977, 99, 8461

13. Epp, D. N. Em The Chemistry of Food Dyes, Sarquis, M., ed.; Palette of Color Monograph Series, Terrific Science Press: Middletown, 1995, p. 11.

14. Timberlake, C. F; Bridle, P. Em The Flavonoids - Part I; Harborne, J. B; Mabry, T. J.; Mabry, H., eds.; Academic Press: New York, 1975, p. 215-224.

15. Skoog, D. A.; West, D. M.; Holler, F. J.; Analytical Chemistry - An Introduction, $6^{\text {th }}$ ed., Saunders: Philadelphia, 1994.

16. Willard, H. H.; Merritt, J. R.; Dean, J. A.; Settle, F. A.; Instrumental Methods of Analysis, $7^{\text {th }}$ ed., Belmont: California, 1988.
17. Underwood, A. L.; J. Chem. Educ. 1954, 31, 394

18. Amdur, S.; Levene, W. J.; J. Chem. Educ. 1974, 51, 136.

19. Godd, R. F.; Hume, D. N.; Anal. Chem. 1954, 26, 1740.

20. Eriksen, S. P.; Connors, K. A.; J. Pharm. Sci. 1964, 53, 465.

21. Goddu, R. F.; Hume, D. N.; Anal. Chem. 1954, 26, 1679.

22. Goddu, R. F.; Hume, D. N.; Anal. Chem. 1954, 26, 1740

23. Marple, T. L.; Hume, D. N.; Anal. Chem. 1956, 28, 1116.

24. Grunwald, E.; Anal. Chem. 1956, 28, 1112.

25. Flaschka, H.; Butcher, J.; Mikrochim. Acta 1964, 2-4, 401.

26. Still, E.; Ringbom, A.; Anal. Chim. Acta 1965, 33, 50.

27. Skrifvars, B.; Ringbom, A.; Anal. Chim. Acta 1966, 36, 105.

28. Flaschka, H.; Speights, R.; Talanta 1968, 15, 1467.

29. Young, M. G.; Clarke, T. H.; Schlick, R. T.; Microchem. J. 1968, 13, 712.

30. Groeneveld, E. R.; Denboef, G.; Analyst 1969, 94, 860.

31. Lalancette, R. A.; Koubek, K. G.; Microchem. J. 1972, 17, 72.

32. Johansson A.; Anal. Chim. Acta 1972, 61, 285.

33. Flaschka, H. A.; Paschal, D. C.; Microchem. J. 1975, 20, 70.

34. Sato, H.; Anal. Chim. Acta 1978, 96, 215.

35. Haartman, C. V.; Talanta 1980, 27, 217.

36. Maleki, N.; Ramezani, Z.; Kaabi, H.; Anal. Chim. Acta 1996, 318, 373.

37. Macca, C.; Anal. Chim. Acta 2002, 456, 313.

38. Macca, C.; Solda, L.; Zancato, M.; Anal. Chim. Acta 2002, 470, 277.

39. El Wahed, M. G. A.; Metwally, S. M.; Mater. Chem. Phys. 2003, 78, 299.

40. Wilhite, R. N.; Underwood, A. L.; Anal. Chem. 1955, 27, 1334.

41. Simonsen, S. H.; Burnett, H. M.; Anal. Chem. 1955, 27, 1336.

42. Shapiro, L.; Brannock, W. W.; Anal. Chem. 1955, 27, 725.

43. Marple, T. L.; Przybylowicz, E. P.; Hume, D. N.; Anal. Chem. 1956, 28, 1892.

44. Flaschka, H.; Butcher, J.; Microchem. J. 1963, 7, 407.

45. Flaschka, H.; Butcher, J.; Analyst 1965, 54, 36.

46. Ringbom, A.; Skrifvars, B.; Still, E.; Anal. Chem. 1967, 39, 1217.

47. Nasouri, F. G.; Shahine, S. A. F.; Magee, R. J.; Microchem. J. 1967, 12, 26.

48. Elghamry, M. T.; Frei, R. W.; Anal. Chem. 1968, 40, 1986.

49. Boef, G. D.; Vanderlinden, W. E.; Beyer, S.; Mikrochim. Acta 1971, 761.

50. Vanderlinden, W. E.; Beijer, S.; Boef, G. D.; Mikrochim. Acta 1972, 334.

51. Denboef, G.; Ozinga, W.; Vanrossum, G. J.; Anal. Chim. Acta 1977, 92, 387.

52. Reilley, C. N.; Schweizer, B.; Anal. Chem. 1953, 25, 1934.

53. Reilley, C. N.; Schweizer, B.; Anal. Chem. 1954, 26, 1124

54. Boyle, W. G.; Robinson, R. J.; Anal. Chem. 1958, 30, 958.

55. Verma, B. C.; Sharma, D. K.; Jamwal, V. S.; Jasrotia, S.; Sambra, B. S.; Indian J. Chem. 1995, 34, 668.

56. Rocha, F. R. P.; Reis, B. F.; J. Chem. Educ. 2000, 77, 258.

57. Beilby, A. L.; Landowsk, C. A.; J. Chem. Educ. 1970, 47, 238.

58. Perrin, D. D.; Dempsey, B.; Buffers for pH and Metal Ion Control, Chapman and Hall: London, 1974

59. Mendham, J.; Dennem, R. C.; Barnes, J. D.; Thomas, M. J. K.; Vogel Análise Química Quantitativa, 6a. ed., LTC: Rio de Janeiro, 2002.

60. Vogel, A. I.; Química Analítica Qualitativa, Mestre Jou: São Paulo, 1981. 